\title{
Reducing microscopy-based malaria misdiagnosis in a low-resource area of Tanzania
}

\author{
LISA K. ALLEN ${ }^{1}$, JENNIFER M. HATFIELD ${ }^{1}$ and MANGE J. MANYAMA ${ }^{2^{*}}$ \\ ${ }^{1}$ Department of Community Health Sciences, Faculty of Medicine, University of Calgary, 3330 Hospital Drive \\ $N W$, Calgary $A B, T_{2} N$ 4N1, Canada \\ ${ }^{2}$ Catholic University of Health and Allied Health Sciences, PO Box 1464, Mwanza, Tanzania
}

\begin{abstract}
Misdiagnosis of malaria is a major problem in Africa leading not only to incorrect individual level treatment, but potentially the acceleration of the spread of drug resistance in low-transmission areas. In this paper we report on the outcomes of a simple intervention that utilized a social entrepreneurship approach (SEA) to reduce misdiagnosis associated with hospital-based microscopy of malaria in a lowtransmission area of rural Tanzania. A pre-post assessment was conducted on patients presenting to the hospital outpatient department with malaria and non-malaria like symptoms in January 2009 (preintervention) and June 2009 (post-intervention). All participants were asked a health seeking behavior questionnaire and blood samples were taken for local and quality control microscopy. Multivariate logistic regression was conducted to determine magnitude of misdiagnosis with local microscopy pre- versus- post intervention. Local microscopy pre-intervention specificity was $29.5 \%(95 \% \mathrm{Cl}=21.6 \%-38.4 \%)$ whereas the post intervention specificity was $68.6 \%(95 \% \mathrm{Cl}=60.2 \%-76.2 \%)$. Both pre and post intervention sensitivity were difficult to determine due to an unexpected low number of true positive cases. The proportion of participants misdiagnosed pre-intervention was $70.2 \%(95 \% \mathrm{Cl}=61.3 \%-78.0 \%)$ as compared to $30.6 \%(95 \% \mathrm{Cl}=$ $23.2 \%-38.8 \%$ ) post-intervention. This resulted in a $39.6 \%$ reduction in misdiagnosis of malaria at the local hospital. The magnitude of misdiagnosis for the pre-intervention participants was $5.3(95 \% \mathrm{Cl}=3.1-9.3)$ that of the post-intervention participants. In conclusion, this study provides evidence that a simple intervention can meaningfully reduce the magnitude of microscopy-based misdiagnosis of malaria for those individuals seeking treatment for uncomplicated malaria. We anticipate that this intervention will facilitate a valuable and sustainable change in malaria diagnosis at the local hospital.
\end{abstract}

Keywords: malaria, microscopy, misdiagnosis, Tanzania

\section{Introduction}

Despite extensive control and elimination efforts, malaria remains a leading cause of morbidity and mortality for children under five (Mboera et al. 2007; WHO 2011). Malaria is strongly associated with poverty as parasite prevalence is known to be higher in poorer populations in rural areas (WHO, 2012). As a compounding effect, rural and remote health facilities are often under-resourced, resulting in a decline in the quality of malaria diagnosis and treatment (Bell et al. 2006). The combination of these two risk factors may create the perfect storm for malaria misdiagnosis and improper case management.

The 2010 World Health Organization (WHO) Guidelines for the Treatment of Malaria recommend presumptive diagnosis and treatment of children under the age of five in endemic areas only when microscopy or malaria rapid diagnostic tests (mRDTs) are unavailable (WHO 2010a). Despite these recommendations, presumptive diagnosis remains a key diagnostic algorithm in low-resource areas due to: lack of quality microscopy or RDTS, patient pressure, fear of missing a malaria case and disregard of negative laboratory tests when available (Chandler et al., 2008). The reliance on presumptive diagnosis has been shown to be associated with considerable misdiagnosis and over-treatment (Gwer et al., 2007; Nankabirwa et al., 2009).

Consequently, communities and individuals in low-resource areas may experience wasted resources on drug purchase, over-medication of children, drug shortages, and unnecessary

\footnotetext{
* Correspondence: Mange Manyama; E-mail: mmajfe@hotmail.com
} 
morbidity and mortality due to improper treatment of the true cause of acute febrile illness (Zuroc et al., 2006; Hume et al., 2008). Over-treatment with artemisinin combination therapies (ACT) is also thought to be a main force driving the spread of Plasmodium drug resistance (Rafael et al., 2006; WHO, 2010b). Drug resistance is one of the most serious problems facing malaria elimination (White et al., 2009). By 2006 Plasmodium falciparum had developed resistance to almost all forms of anti-malarial drugs available except for artemisinin derivatives and this has since emerged in Cambodia (Noedl et al., 2008; Dondorp et al., (2009).

Furthermore, the literature pertaining to Tanzania consistently demonstrates that the high variability of hospital records and the lack of standard operating procedures (SOPs) does not provide a clear picture of malaria epidemiology (Shears, 2000; Snow et al., 2005; Maegga et al., 2005; Masika et al., 2006; Makundi et al. 2007). There is an inevitable need to improve upon existing malaria laboratory diagnostics and disease surveillance techniques to promote better management of malaria (Maegga et al., 2005; Makundi et al., 2007; WHO 2010a). In order to address these salient issues it is crucial that rural, low-resource health facilities have access to accurate and reliable microscopy or mRDTs. Here, we report the impact of a simple intervention aimed to improve the accuracy of malaria microscopy that was implemented in a rural lowresource and low-transmission area of northern Tanzania. This intervention provides evidence that simple changes to improve the quality of malaria microscopy can provide foundations for substantial decreases in misdiagnosis.

\section{Materials and Methods}

\section{Study location}

The study was conducted at a hospital in the Ngorongoro Conservation Area (NCA) of the northern Tanzanian highlands. It is the only hospital serving a population of approximately 51,000 people who are predominantly Maasai pastoralists. Elevation ranges from $1000 \mathrm{~m}$ to over $3000 \mathrm{~m}$ above sea level, thus placing the population in a low transmission zone for malaria (Chandler et al., 2006). Based on a formative assessment of hospital out-patient records in 2008, the proportion of patients presenting with febrile symptoms who were diagnosed as positive for malaria based on microscopy was over $40 \%$ (Allen et al., 2010). Although this is not a true estimate of malaria prevalence for the area, one may anticipate from the regional relationship between malaria prevalence and altitude that this proportion should be much lower (Chandler et al., 2006), suggesting a high level of malaria misdiagnosis and over-treatment with anti-malarial drugs may be occurring.

\section{Intervention description}

Sustainability is a key to successful health interventions. We applied a social entrepreneurship approach (SEA) to foster a sustainable application of the research. The guiding theory and approach to this intervention has been described in detail elsewhere (Allen et al., 2010). The intervention was conducted in May 2009 and consisted of 4 major components: (1) training of local laboratory staff to use WHO standard operating procedures (SOPs) and implementation of the SOPs; (2) addition of an institutionally trained laboratory technologist to the local laboratory; (3) training on local epidemiological information regarding expected prevalence of malaria in this low transmission zone; and (4) feedback on current levels of malaria microscopy sensitivity, specificity and misdiagnosis following baseline data collection in January 2009 (pre-intervention). Following the intervention, a post-intervention data collection period (June 2009) was conducted to assess changes in sensitivity, specificity and misdiagnosis magnitude as measured by the odds ratio of malaria misdiagnosis for microscopy-based detection of malaria at the local hospital. 


\section{Study design and sample size}

A cross-sectional study with sampling occurring in January (pre-intervention) and June of 2009 (post-intervention) was performed. During both of the sampling times, the region received limited rainfall. Study design was based on the WHO Evaluation of diagnostic tests for infectious diseases: general principles (Banoo et al., 2006) and the WHO Evaluation of rapid diagnostic tests: malaria (Bell \& Peeling, 2006). A demographic and health seeking behavior (HSB) questionnaire was administered in parallel to participants in all study groups in order to determine if patient level factors confound or modify the odds of misdiagnosis.

Sample size was calculated based on an expected malaria prevalence of 9.3\% (Chandler et al., 2006), a 95\% confidence and precision level of $5 \%$. The simple formula of $n=Z^{2} P(1-P) / d^{2}$, where $n=$ sample size, $Z=Z$ statistic for a level of confidence, $P=$ expected prevalence or proportion and $d$ = precision was used for calculations (Naing, 2006). The resulting total sample size required per group (pre and post intervention) was $n=129$.

\section{Inclusion and exclusion criteria of study participants}

Inclusion criteria: Patients presenting to the local hospital who lived in the NCA and gave informed consent that met the following inclusion criteria were enrolled: 1 ) clinical suspicion of malaria sent for microscopy requisition; and 2) non-malaria like symptoms sent to the laboratory for other diagnostic tests. The second group of participants was deemed important due to the short timeframe for sampling paired with the limited number of patients presenting to the local hospital each day. During data analysis, no distinctions were made between those with clinical suspicion and those without. Age of participants was not used as an inclusion criteria, but rather to dichotomize the two groups in to $\leq 5$ years and $>5$ years (actual age was not recorded).

Exclusion criteria: Any patients with known or suspected pregnancy, patients with severe and/or complicated malaria, and patients with severe and complicated co-morbidities as defined by the clinical health officer.

\section{Sampling strategy}

Research personnel, together with clinical officers at the local hospital identified all potential participants presenting to the outpatient department within the data collection time frame. Patients presenting to the outpatient department were assessed to determine if they met the inclusion criteria by both the clinical officer and the research personnel. All individuals meeting the inclusion criteria were asked for informed consent before conducting the HSB questionnaire and laboratory microscopy. The same sampling strategy was used for both pre and post intervention participant recruitment.

\section{Local microscopy and quality control}

In the laboratory, blood was obtained by a finger prick; a thick and thin blood smears were prepared according to standard WHO procedures (WHO, 2010b). One of the laboratory technicians working in the hospital laboratory stained slides with either Fields stain or Giemsa and estimated parasites density against 200 white blood cells. At least 100 fields on each slide were examined before the slide being declared negative for malaria parasites (WHO, 2010b). Treatment of patients for malaria was based on the results from local microscopy.

Blood slides were stored in a cool and dark location to maintain sample integrity until being sent to Catholic University of Health and Allied Health Sciences (CUHAS) in Mwanza, Tanzania to be read by two independent laboratory technologists. These quality control (QC) laboratory technologists were blinded to each other as well as the local hospital microscopy results. QC Laboratory technologists at CUHAS held a 3-year diploma in laboratory techniques with more than 6 years working experience in a parasitology laboratory at the time of the study. In addition, both the local laboratory technicians and QC laboratory technologists had been 
attending refresher courses to improve their skills in malaria microscopy. At both sites, the WHO standard operating procedures for microscopy were followed (WHO, 2010b).

After completion of standard WHO microscopy procedures, a blood smear was considered to be either a true positive or true negative only when both external CUHAS QC readers agreed on the result. External CUHAS QC readers were blinded to the microscopy results of the local hospital laboratory as well as to each other. Percent agreement and kappa values were used for analysis for both agreement between the external CUHAS QC readers and agreement between external CUHAS QC and the local hospital laboratory (Landis \& Koch 1977). Parasitemia was not considered during evaluation of true positives or true negatives, only that both CUHAS QC readers identified the slide as either positive or negative (binary result). Parasitemia was used as a measure of infection severity for clinical purposes but was not taken into account during QC measures.

\section{Data analysis}

All data were entered into Microsoft Excel version 12.3.3 and analysis was performed using STATA version 11.0. Study participants with missing microscopy data were excluded from analysis. Sensitivity and specificity with 95\% confidence intervals were calculated using standard procedures in STATA. Misdiagnosis by local microscopy was recorded when there was a disagreement with the external CUHAS QC result and the local hospital test result. Logistic regression model was used to determine the magnitude of misdiagnosis during both preintervention and post-intervention. Potential effect modifiers or confounders such as age and gender were included in the multivariate analysis. Participants were stratified into $>5$ year and $\leq 5$ years to develop binary categories for age based on WHO guidelines for the presumptive treatment of malaria in children under 5 years of age.

\section{Ethical considerations}

Ethical approval for this study was granted from the Conjoint Health Research Ethics Board (CHREB) at the University of Calgary and the Medical Research Coordinating Committee of the National Institute of Medical Research in Tanzania. In Tanzania the Commission on Science and Technology approved a research permit. Informed consent was obtained from all study participants. The informed consent form was translated into Kiswahili and was then further translated into the local language of Maa for those participants unable to understand Kiswahili. For all participants who were able to provide a signature, written consent was obtained. For those participants who were unable to provide a written signature, verbal consent was obtained by the clinical officers and "verbal consent" was noted on the signature line of the consent form. Informed consent for study participants under the age of 5 was provided by a parent, guardian or next of kin. For children between the ages of 5 to 16 years of age their informed assent together with parental/guardian or next of kin informed consent was obtained.

\section{Results}

The actual sample sizes for the pre and post intervention groups' varied marginally from the original calculations due to logistics of data collection (pre $n=124$ and post $n=144$ ). Throughout the course of the study, a limited number of patients with malaria-like symptoms declined consent to participate (<10 patients). Characteristics including estimates of local hospital microscopy positive tests, quality control (QC) microscopy true positives, local hospital microscopy sensitivity and the proportion of patients misdiagnosed at the local hospital are summarized in Table 1.

Local microscopy pre-intervention specificity was $29.5 \%(95 \% \mathrm{Cl}=21.6 \%-38.4 \%)$ whereas the post intervention specificity was $68.6 \%(95 \% \mathrm{Cl}=60.2 \%-76.2 \%)$. Both pre and post intervention sensitivity were difficult to determine due to an unexpected low number of true positive cases. 
The proportion of participants misdiagnosed pre-intervention was $70.2 \%(95 \% \mathrm{Cl}=61.3 \%-78.0 \%)$ as compared to $30.6 \%(95 \% \mathrm{Cl}=23.2 \%-38.8 \%)$ post-intervention. This resulted in a $39.6 \%$ reduction in misdiagnosis of malaria at the local hospital. The magnitude of misdiagnosis for the preintervention participants was $5.3(95 \% \mathrm{Cl}=3.1-9.3)$ that of the post-intervention participants (Table 1).

Table 1: Characteristics of the participant groups both pre and post intervention

\begin{tabular}{lll}
\hline Characteristic & Pre $(95 \% \mathrm{Cl})$ & Post $(95 \% \mathrm{Cl})$ \\
\hline $\mathrm{N}$ & 124 & 144 \\
$\leq 5$ years & $36.3 \%(27.8 \%-45.4 \%)$ & $37.5 \%(29.6 \%-45.9 \%)$ \\
Male & $47.6 \%(38.5 \%-56.7 \%)$ & $50.7 \%(42.2 \%-59.1 \%)$ \\
Local hospital microscopy positives & $70.9 \%(62.1 \%-78.8 \%)$ & $32.6 \%(25.0 \%-40.9 \%$ \\
QC microscopy true positives & $1.6 \%(0.2 \%-5.7 \%)$ & $2.8 \%(0.8 \%-6.9 \%)$ \\
Misdiagnosis at local hospital & $70.2 \%(61.3 \%-78.0 \%)$ & $30.6 \%(23.2 \%-38.8 \%)$ \\
Local hospital microscopy sensitivity ${ }^{*}$ & $100 \%(15.8 \%-100 \%)$ & $75 \%(19.4 \%-99.4 \%)$ \\
Local hospital microscopy specificity & $29.5 \%(21.6 \%-38.4 \%)$ & $68.6 \%(60.2 \%-76.2 \%)$ \\
\hline
\end{tabular}

*Accuracy of the sensitivity estimate is reduced due to lower than anticipated true positive cases of malaria at the local hospital (2/124 pre-intervention and 4/144 post intervention).

The crude odds ratio was used to describe the magnitude of misdiagnosis for patients with malaria-like symptoms as compared pre- and post-intervention. Logistic regression model was used to determine the magnitude of misdiagnosis during both pre-intervention and postintervention. Potential effect modifiers or confounders such as age and gender were included in the multivariate analysis (Table 2).

Table 2: Age group stratified analysis of the odds of misdiagnosis pre intervention as compared to post intervention

\begin{tabular}{l|l}
\hline Age Group & Odds Ratio $(95 \% \mathrm{Cl})$ \\
\hline$\leq 5$ years & $11.9(3.9-39.9)$ \\
$>5$ years & $4.0(2.0-8.1)$ \\
Crude & $5.3(3.1-9.3)$ \\
M-H combined * & $5.6(3.2-9.6)$ \\
\hline
\end{tabular}

* M-H Test of Homogeneity p-value $=0.077$

When comparing the two external CUHAS QC readers using the entire data set $(n=268)$, there was a $97.39 \%$ inter-rater agreement $(k=0.6181, \mathrm{SE}=0.0610)$. The results from local hospital laboratory microscopy had $51.12 \%$ agreement $(\mathrm{k}=0.0291$, SE $=0.0230)$ with CUHAS QC reader \#1 and $52.24 \%$ agreement $(k=0.0514, \mathrm{SE}=0.0219)$ with CUHAS QC reader \#2. The low level of agreement between the local hospital laboratory microscopy and the external CUHAS QC readers is due to a high number of false positives diagnosed in the local laboratory.

The proportion of participants diagnosed as positive by the local hospital laboratory during pre-and post-intervention assessments are presented in Table 1. The intervention can be associated with an approximately a $36.2 \%$ decrease in positive malaria microscopy-based diagnoses at the local hospital.

Based on comparisons with the CUHAS QC results, changes in sensitivity and specificity from pre to post intervention are reported in Table 1. Estimates of sensitivity were calculated using an unexpectedly low number of true positives (pre intervention true positives $n=2$, post intervention true positives $\mathrm{n}=4)$. The $75 \%(95 \% \mathrm{Cl}=19.41 \%-99.37 \%)$ sensitivity for post intervention represents the local laboratory staff identifying 3 out of 4 true positives.

There was no statistically significant confounding or modifying effects of age group or gender on misdiagnosis in the multivariate logistic regression models. Therefore the odds of misdiagnosis for the pre-intervention participants were 5.34 (3.07-9.34) the odds of misdiagnosis for the post-intervention participants. 


\section{Discussion}

It is widely recognized that accurate laboratory-based diagnosis of malaria is central to guiding proper clinical decisions and reducing the use of unnecessary antimalarials (WHO 2004; Wongsrichanalai et al., 2007). Despite this fact, the quality of malaria diagnosis at health care facilities in rural Tanzania is generally poor due to limited skill of laboratory personnel and a lack of essential supplies (Ishengoma et al., 2010). Sustainable improvements in the accuracy of malaria microscopy at the local level are challenging often due to poor blood film preparation and a lack of expertise in slide review (Wongsrichanalai et al., 2007), but as we have shown through this simple intervention, reductions in misdiagnosis for all age groups is achievable.

We anticipate that the reduction in the magnitude of misdiagnosis demonstrated by this intervention will ultimately improve patient care and maximizing the cost to benefit ratio. But, we must be mindful that improvements at the laboratory level, do not always translate directly to clinical decision-making. Previous research in Tanzania has shown that, presumptive diagnosis of malaria at the clinical level has been associated with a disregard for negative microscopy results, patient pressure and preference, clinician disease preference and peer pressure (Chandler et al., 2008; Leslie et al., 2012). Therefore it is imperative that clinicians are included in the quality improvement process and efforts to develop a culture of malaria diagnosis that does not rely solely on presumptive diagnosis and treatment are made.

Quality laboratory-based diagnosis, paired with congruent clinical decision-making will provide a comprehensive picture of the epidemiology of malaria as well as the efficacy of, and potential resistance to, drug therapies (Sheers, 2000; Makundi et al., 2007). Accurate disease profiling could lead to programme modifications that optimize the control of malaria and properly guide funding allocation. Additionally, several areas in Tanzania are prone to malaria outbreaks because of unstable malaria transmission, which catch several communities off-guard further exacerbating already high morbidities and mortalities (Mboera \& Kitua 2001). Early confirmation of malaria as the cause of a febrile illness outbreak through accurate diagnostic methods, in addition to the development of a management strategy, could better prepare communities for outbreaks. The improvements in malaria diagnosis achieved at this rural hospital though our simple intervention demonstrates that it is possible to reduce malaria misdiagnosis and ultimately begin to improve patient care and generate more accurate epidemiology profiles for this low-transmission and low-resource area of Tanzania.

This study had a number of limitations. Due to the small number of true positives it is difficult to reliably judge the effect of the intervention on sensitivity. The observed decrease in sensitivity was due to the local laboratory technicians failing to detect a single true positive case. Increasing sample size would assist in correcting for wide confidence intervals and the resulting large margin of error for sensitivity measurements. Yet, the current sample size does provide reliable estimates of specificity as well as odds ratios for misdiagnosis pre versus post intervention.

Although we report the crude odds ratio for malaria misdiagnosis, and not the age stratified odds ratios in this case (due to a non-significant $M-H$ test of homogeneity $p=0.0774$ ), it is important to discuss the difference between statistical significance and clinical importance (Altman \& Bland, 1996). The finding of statistical non-significance may have been influenced by sample size that was not sufficient to detect a significant difference between the two age groups ( $95 \% \mathrm{Cl}$ are very wide for the odds ratio estimates). Despite this, the finding that there may be a clinically important difference in the odds of misdiagnosis for those under the age of five is salient. In areas of low transmission, a mindset of "safer than sorry" may result in an increase in malaria misdiagnosis for children under the age of five (Nankabirwa et al., 2009). We support the idea that clinicians and laboratory personnel should be mindful of presumptively diagnosing malaria for all patients in this context (D'Acremont et al., 2007). 
In conclusion, reducing malaria misdiagnosis is a key component of elimination and control efforts in low-transmission areas of Tanzania. This study provides evidence that a simple intervention can meaningfully reduce the magnitude of microscopy-based malaria misdiagnosis for those individuals seeking treatment for uncomplicated malaria. We anticipate that this intervention, together with congruent changes in the culture of clinical decision-making, will facilitate a valuable and sustainable change in malaria diagnosis and treatment for this population.

\section{Competing Interests}

The authors declare no competing interests.

\section{Authors Contributions}

LKA was responsible for designing and implementing the field research, analyzing the data and writing the manuscript. JMH was responsible for overseeing the field research and editing the final manuscript. $M M$ was responsible for designing and implementing the field research and editing the final manuscript. All authors approved the final manuscript.

\section{Acknowledgments}

We thank Dr. Nicholas Adams, Dr. Yadira Roggeveen, Eresto Medard, Mameso Frederick, Grace Mutel, Bahati Medard, Rita Maley, John Riverian and Sylivery Sylivester for their collaboration and provision of quality control services. We are also grateful to Jeremy Ho and Giselle DeVetten at the University of Calgary for assistance in the field. This study received financial assistance from the University of Calgary and the Bachelor of Health Sciences Program. Lisa K. Allen was funded as a Vanier Canada Graduate Scholar for her PhD dissertation.

\section{References}

Allen, L.K., Hetherington, E., Manyama, M., Hatfield, J.M. \& van Marle, G. (2010) Using the social entrepreneurship approach to generate innovative and sustainable malaria diagnosis interventions in Tanzania: a case study. Malaria Journal 9:42.

Altman, D.G. \& Bland, J.M. (1996) Absence of evidence is not evidence of absence. Australian Veterinary Journal 74, 311.

Banoo, S., Bell, D., Bossuyt, P., Herring, A., Mabey, D., Poole, F., Smith, P.G., Sriram, N., Wongsrichanalai, C., Linke, R., O'Brien, R., Perkins, M., Cunningham, J., Matsoso, P., Nathanson, C.M., Olliaro, P., Peeling, R.W., Ramsay, A; TDR Diagnostic Evaluation Expert Panel. (2006) Evaluation of diagnostic tests for infectious diseases: general principles. Nature Review Microbiology 4 (Suppl 12), S20-32.

Bell, D. \& Peeling, R.W. (2006) Evaluation of rapid diagnostic tests: malaria. Nature Review Microbiology 4 (Suppl 9), S34-38.

Bell, D., Wongsrichanalai, C. \& Barnwell, J.W. (2006) Ensuring quality and access for malaria diagnosis: how can it be achieved? Nature Review Microbiology 4 (Suppl 9), S7-20.

Chandler, C.I., Drakeley, C.J., Reyburn, H. \& Carneiro, I. (2006) The effect of altitude on parasite density case definitions for malaria in northeastern Tanzania. Tropical Medicine and International Health 11, 1178-1184.

Chandler, C.I., Jones, C., Boniface, G., Juma, K., Reyburn, H. \& Whitty, C.J. (2008) Guidelines and mindlines: why do clinical staff over-diagnose malaria in Tanzania? A qualitative study. Malaria Journal 7:53. 
D'Acremont, V., Lengeler, C. \& Genton, B. (2007) Stop ambiguous messages on malaria diagnosis. BMJ 334, 489.

Dondorp, A.M., Nosten, F., Yi, P., Das, D., Phyo, A.P., Tarning, J., Lwin, K.M., Ariey, F., Hanpithakpong, W., Lee, S.J., Ringwald, P., Silamut, K., Imwong, M., Chotivanich, K., Lim, P, Herdam, T., An, S.S., Yeung, S., Singhasivanon, P, Day, N.P., Lindegardh, N., Socheat, D. \& White, N.J. (2009) Artemisinin resistance in Plasmodium falciparum malaria. New England Journal of Medicine 361, 455-467.

Gwer, S., Newton, C.R. \& Berkley, J.A. (2007) Over-diagnosis and co-morbidity of severe malaria in African children: a guide for clinicians. American Journal of Tropical Medicine and Hygiene 77 (Suppl 6), 6-13.

Hume, J.C., Barnish, G., Mangal, T., Armazio, L., Streat, E. \& Bates, I. (2008) Household cost of malaria overdiagnosis in rural Mozambique. Malaria Journal 7:33.

Ishengoma, D.R., Derua, Y.A., Rwegoshora, R.T., Tenu, F., Massaga, J.J., Mboera, L.E.G., \& Magesa, S.M. (2010) The performance of health laboratories and the quality of malaria diagnosis in six districts of Tanzania. Annals of Tropical Medicien and Parasitology 104, 123135.

Kahama-Maro, J., D'Acremont, V., Mtasiwa, D., Genton, B. \& Lengeler, C. (2011) Low quality of routine microscopy for malaria at different levels of the health system in Dar es Salaam. Malaria Journal 10:332.

Landis, J.R. \& Koch, G.G. (1977) The measurement of observer agreement for categorical data. Biometrics 33, 159-174.

Leslie, T., Mikhail, A., Mayan, I., Anwar, M., Bakhtash, S., Nader, M., Chandler, C., Whitty, C.J. \& Rowland, M. (2012) Overdiagnosis and mistreatment of malaria among febrile patients at primary healthcare level in Afghanistan: observational study. BMJ 345:e4389.

Maegga, B.T., Cox, J. \& Malley, K.D. (2005) Malaria in the southern highlands of Tanzania: a review of hospital records. Tanzan Health Research Bulletin 7, 125-132.

Makundi, E.A., Mboera, L.E.G., Malebo, H.M. \& Kitua, A.Y. (2007) Priority setting on malaria interventions in Tanzania: strategies and challenges to mitigate against the intolerable burden. American Journal of Tropical Medicine and Hygiene 77 (Suppl 6), 106-111.

Masika, P.M., Semarundu, W.J., Urassa, R., Mosha, J., Chandramohan, D. \& Gosling, R.D. (2006) Over-diagnosis of malaria is not a lost cause. Malaria Journal 5:120.

Mboera, L.E.G. \& Kitua, A.Y. (2001) Malaria epidemics in Tanzania: An overview. African Journal of Health Sciences 8, 17-23.

Mboera, L.E.G., Makundi, E.A. \& Kitua, A.Y. (2007) Uncertainty in malaria control in Tanzania: crossroads and challenges for future interventions. American Journal of Tropical Medicine and Hygiene 77, (Suppl 6), 112-118.

Naing, L., Winn, T. \& Rusli, B. (2006) Practical issues in calculating the sample size for prevalence studies. Medical Statistics 1:9-14.

Nankabirwa, J., Zurovac, D., Njogu, J.N., Rwakimari, J.B., Counihan, H., Snow, R.W. \& Tibenderana, J.K. (2009) Malaria misdiagnosis in Uganda--implications for policy change. Malaria Journal 8:66.

Noedl, H., Se, Y., Schaecher, K., Smith, B.L., Socheat, D. \& Fukuda, M.M. (2008) Evidence of artemisinin-resistant malaria in western Cambodia. New England Journal of Medicine 359, 2619-2620.

Rafael, M.E., Taylor, T., Magill, A., Lim, Y.W., Girosi, F. \& Allan, R. (2006) Reducing the burden of childhood malaria in Africa: the role of improved. Nature 444 (Suppl 1), 39-48.

Shears, P. (2000) Emerging and reemerging infections in africa: the need for improved laboratory services and disease surveillance. Microbes Infection 2, 489-495.

Snow, R.W., Guerra, C.A., Noor, A.M., Myint, H.Y. \& Hay, S.I. (2005) The global distribution of clinical episodes of Plasmodium falciparum malaria. Nature 434, 214-217. 
White, N.J., Pongtavornpinyo, W., Maude, R.J., Saralamba, S., Aguas, R., Stepniewska, K., Lee, S.J., Dondorp, A.M., White, L.J. \& Day, N.P. (2009) Hyperparasitaemia and low dosing are an important source of anti-malarial drug resistance. Malaria Journal 8:253.

WHO (2004) The role of laboratory diagnosis to support malaria disease management: Focus on the use of rapid diagnostic tests in areas of high transmission. Report of a WHO Technical Consultation: 25-26.

WHO (2010b) Global Report on Antimalarial Drug Efficacy and Drug Resistance: 2000--2010.121.

WHO (2010a) World Health Organization Guidelines for the Treatment of Malaria. Second Edition.

WHO (2011) World Malaria Report. World Health Organization, Geneva, Switzerland.

WHO (2012) World Malaria Report. World Health Organization, Geneva, Switzerland.

Wongsrichanalai, C., Barcus, M.J., Muth, S., Sutamihardja, A. \& Wernsdorfer, W.H. (2007) A review of malaria diagnostic tools: microscopy and rapid diagnostic test (RDT). American Journal of Tropical Medicine Hygiene 77 (Suppl 6), 119-127.

Zurovac, D., Larson, B.A., Akhwale, W. \& Snow, R.W. (2006) The financial and clinical implications of adult malaria diagnosis using microscopy in Kenya. Tropical Medicine and International Health 11, 1185-1194. 